\title{
Mortalidade e incidência da infecção por SARS-CoV-2 (COVID-19) em pacientes internados e operados por fratura de quadril durante a pandemia de SARS-CoV-2 em um hospital de Londres*
}

\section{Mortality and Incidence Rate of SARS-CoV-2 (COVID-19) Infection in Patients Admitted and Operated for Hip Fracture during SARS-CoV-2 pandemic in a London Hospital}

\author{
Faisal Younis Shah ${ }^{1}$ Jaskiran Gill ${ }^{1}$ Hamza Sheikh ${ }^{1}$ Samantha Tross ${ }^{1}$ () \\ ${ }^{1}$ Ealing Hospital, Londres, Reino Unido \\ Endereço para correspondência Faisal Younis Shah, MRCS, 348, \\ Rev Bras Ortop 2021;56(5):594-600. \\ Wokingham Road, Earley, Reading RG67DE, United Kingdom \\ (e-mail: faisalyounisshah@gmail.com).
}

\begin{abstract}
Resumo
Objetivo Estamos fazendo um estudo em pacientes internados com fraturas de quadril para determinar o impacto da COVID-19 no grupo de indivíduos vulneráveis do Reino Unido. O presente estudo nos ajudará a tomar decisões informadas sobre o reinício dos serviços cirúrgicos eletivos e a expansão dos serviços cirúrgicos em casos de traumatismos. Os objetivos do presente estudo são a determinação da incidência de COVID-19 em pacientes internados com fratura de quadril e 1) determinar a mortalidade em 30 dias em pacientes com fratura de quadril submetidos ao tratamento cirúrgico; 2) determinar a mortalidade em 30 dias de pacientes com fratura de quadril e COVID-19; 3) comparar esses dados com a mortalidade associada a fraturas de quadril em anos anteriores.

Métodos Este é um estudo de coorte unicêntrico, observacional e retrospectivo com 65 pacientes com fraturas de quadril internados em nossa instituição. Além dos dados epidemiológicos, os prontuários dos pacientes foram acompanhados por 14 dias

Palavras-chave

- COVID-19

- fratura de quadril

- mortalidade

- infecções

- risco quanto a resultados positivos para COVID-19 à reação em cadeia de polimerase (PCR, na sigla em inglês) em amostras de swab, e por 30 dias quanto à mortalidade. Resultados Na nossa amostra, 64\% dos pacientes não apresentavam comorbidade significativa. A incidência de infecções nosocomiais por COVID-19 foi de 9\%. A mortalidade geral em 30 dias foi de $15 \%$. A mortalidade foi muito maior em pacientes COVID-positivos (40\%) e em pacientes com "risco muito alto" (63\%) operados durante este período.
\end{abstract}

* Estudo desenvolvido no Ealing Hospital, Londres, Reino Unido.

recebido

17 de Agosto de 2020

aceito

28 de Outubro de 2020

Publicado on-line

Março 30, 2021
DOI https://doi.org/

10.1055/s-0041-1726063.

ISSN 0102-3616.
(C) 2021. Sociedade Brasileira de Ortopedia e Traumatologia. All rights reserved.

This is an open access article published by Thieme under the terms of the Creative Commons Attribution-NonDerivative-NonCommercial-License, permitting copying and reproduction so long as the original work is given appropriate credit. Contents may not be used for commercial purposes, or adapted, remixed, transformed or built upon. (https://creativecommons.org/ licenses/by-nc-nd/4.0/)

Thieme Revinter Publicações Ltda., Rua do Matoso 170, Rio de Janeiro, RJ, CEP 20270-135, Brazil 
Conclusão A realização de cirurgias eletivas em pacientes com risco baixo, moderado e alto parece ser segura, já que não houve aumento apreciável da mortalidade. Mais dados são necessários para entender o impacto da COVID-19 em pacientes de risco muito alto.

Abstract

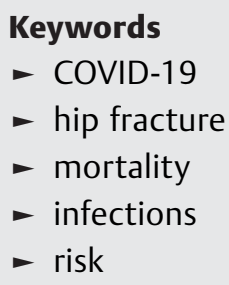

Objective We are doing a study on patients admitted with hip fractures to determine the impact of COVID-19 on a vulnerable patients group in the United Kingdom. This will help us in making informed decisions about restarting elective surgical services and expanding trauma surgical services. The objectives of the study are: 1) to find the incidence of COVID-19 in patients admitted with hip fractures; 2 ) To find the 30-day mortality in patients operated with hip fractures; 3 ) To find the 30-day mortality of patients with hip fracture and COVID 19; and 4) to compare this data with the mortality in hip fractures in previous years.

Methods This is a single-center, observational, retrospective, cohort study involving 65 patients who were admitted in our trust with hip fractures. Besides epidemiological data, patient records were followed-up for 14 days to look for COVID positive polymerase chain reaction (PCR) swabs, and the patient records were followed-up for 30 days to look for mortality.

Results A total of $64 \%$ of the patients had no significant comorbidity. The incidence of hospital-acquired COVID-19 infections in our trust was 9\%. Overall, the 30-day mortality was of $15 \%$. Mortality was much higher in COVID positive patients (40\%) and in patients with "very high risk" (63\%) operated during this period.

Conclusion It should be safe to start elective surgery in patients with low, moderate and high risk without an appreciable rise in mortality. We will need more data to understand the impact of COVID-19 on very high risk patients.

\section{Introdução}

Em dezembro de 2019, uma pandemia de coronavírus começou em Wuhan, China, e se espalhou rapidamente pela maior parte do mundo nos meses seguintes. ${ }^{1}$ A doença foi chamada de "doença por coronavírus 2019" (COVID-19, do inglês coronavirus disease 2019) ou, mais precisamente, de "síndrome respiratória aguda grave por coronavírus 2" (SARS-CoV-2, do inglês severe acute respiratory syndrome - coronavirus 2$)^{2}{ }^{2}$

No Reino Unido, os primeiros casos de coronavírus foram confirmados em 29 de janeiro de 2020. ${ }^{3}$ Em 11 de março de 2020, a Organização Mundial da Saúde (OMS) declarou a doença uma pandemia. ${ }^{4}$ Em 23 de março de 2020, o Reino Unido entrou em lockdown (protocolo de isolamento que geralmente impede o movimento de pessoas ou cargas). ${ }^{5}$ Naquela época, havia 6.650 casos confirmados e 335 mortes (- Fig. 1). No Reino Unido, o pico/platô da doença foi atingido em abril (-Fig. 2). ${ }^{6}$

Durante o lockdown, a maioria dos hospitais no Reino Unido interrompeu todas as cirurgias eletivas para proteger os pacientes (muitos dos quais são idosos e apresentam múltiplas comorbidades) de infecções nosocomiais. Isso também permitiu a liberação de recursos em preparação para o aumento repentino e esmagador de pacientes com COVID-19. No entanto, as cirurgias de emergência e trauma continuaram. Isso foi facilitado pela organização preparatória da equipe e modificação das áreas de atendimento. ${ }^{7} \mathrm{~A}$ interrupção das cirurgias eletivas no Reino Unido gerou um grande acúmulo de pacientes que aguardavam os procedimentos. $^{8}$

A British Orthopaedic Association (BOA, na sigla em inglês) publicou diretrizes sobre o reinício dos atendimentos de casos não urgentes de traumatismo e ortopedia em 15 de maio de $2020 .{ }^{9}$ Nesta publicação, a BOA menciona o estudo com 34 pacientes submetidos a cirurgias eletivas e que desenvolveram sintomas de COVID-19 no período pós-operatório em Wuhan, publicado por Lei et al. ${ }^{10}$ em 2020. Neste estudo, a mortalidade foi de $20,5 \%$ em procedimentos de "categoria 3", como artroplastia total de quadril. Este estudo também relatou taxas de $65 \%$ de internação em unidade de terapia intensiva (UTI) e de 35\% de mortalidade.

Esses números são preocupantes e motivaram a realização de um estudo no Reino Unido para determinar o impacto da COVID-19 neste grupo de pacientes vulneráveis submetidos a cirurgia. Isso nos ajudaria a tomar decisões informadas sobre o reinício de serviços cirúrgicos eletivos e a expansão dos atendimentos de traumas.

$O$ presente estudo foi realizado com pacientes com fraturas de quadril submetidos a cirurgia neste período. Os pacientes com fraturas de quadril são um grupo vulnerável devido à idade e à presença usual de múltiplas comorbidades; esta vulnerabilidade aumentou durante a pandemia. ${ }^{11}$

Cerca de 76.000 fraturas de quadril ocorrem em uma população de 66,46 milhões de residentes no Reino Unido 


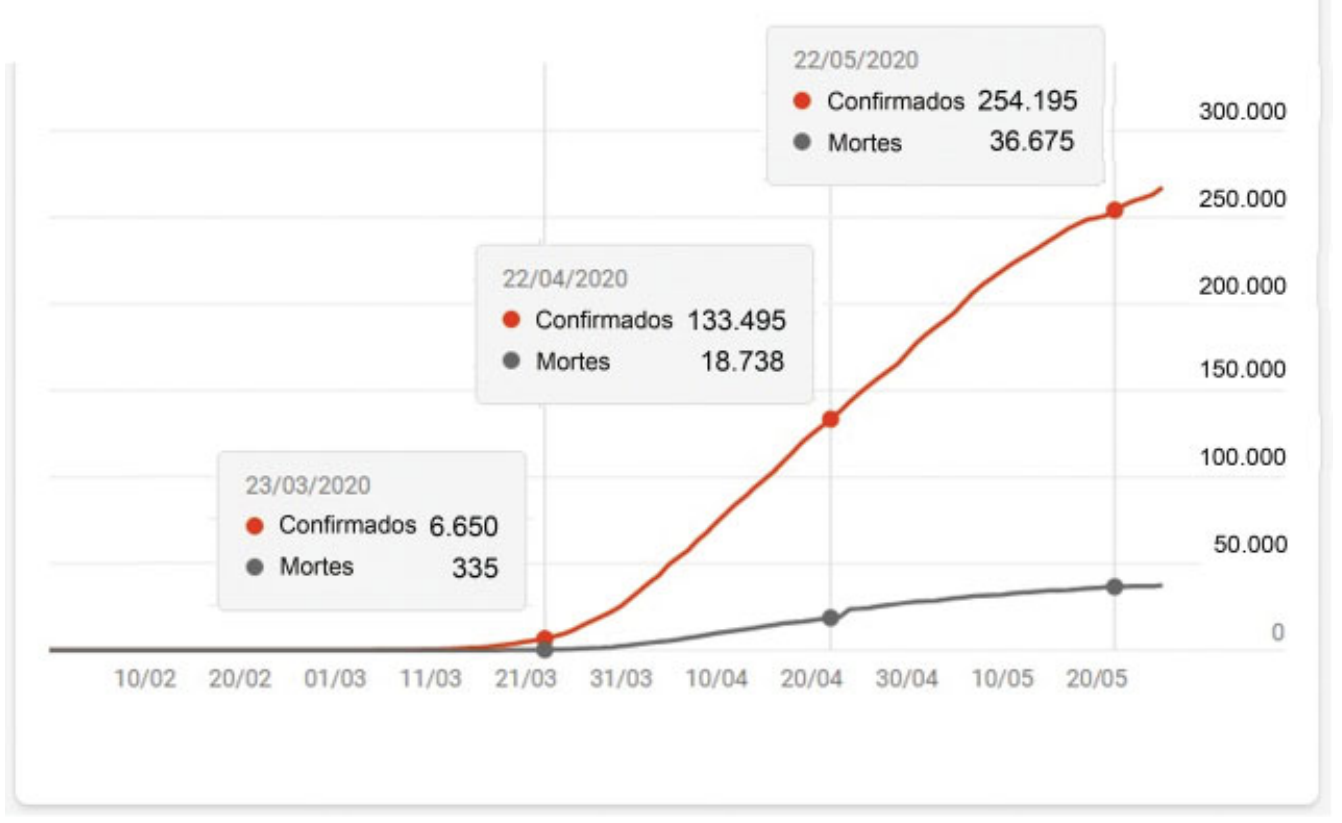

Fig. 1 Número cumulativo de casos e mortes de COVID-19 no Reino Unido.

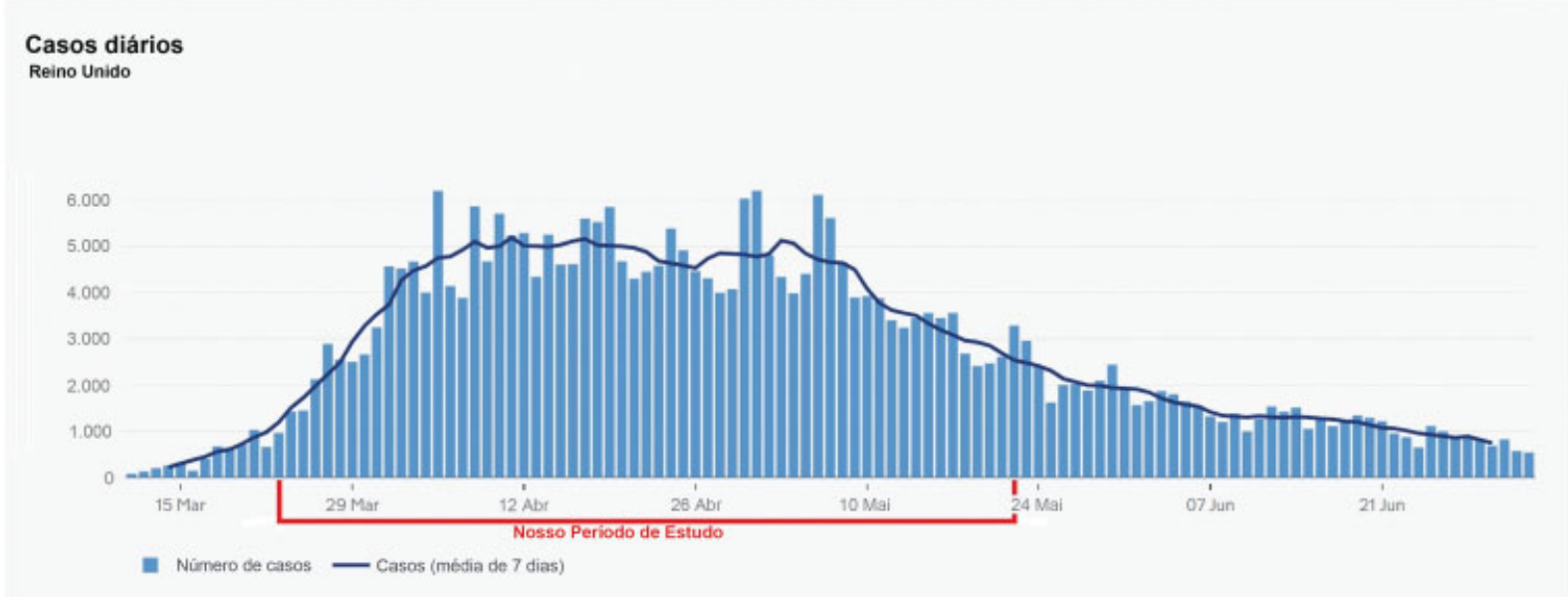

Fig. 2 Incidência diária de casos no Reino Unido.

a cada ano ( 115 por 100.000 por ano). ${ }^{12}$ As fraturas de quadril são comuns em pacientes que já apresentam comorbidades médicas; ${ }^{13}$ por isso, a taxa de mortalidade associada às fraturas de quadril é alta, mesmo na ausência de COVID19. No Reino Unido, as fraturas do fêmur proximal estão associadas a uma mortalidade de $6,2 \%$ nos primeiros 30 $\operatorname{dias}^{14}$ e a uma taxa de mortalidade de $22 \%$ durante o $1^{\circ}$ ano em todo o mundo. ${ }^{15}$ Pacientes idosos com fratura de quadril também apresentam maior risco de morte em curto prazo (entre 22 e $30 \%$ ) em caso de COVID-19. ${ }^{16,17}$ Os pacientes hospitalizados entram em contato com outros pacientes e funcionários e, portanto, apresentam maior risco de desenvolvimento de COVID-19.
Isso, infelizmente, torna este grupo de pacientes ideal para nosso estudo.

O objetivo do nosso estudo observacional é:

1. Determinar a incidência de COVID-19 nosocomial em pacientes com fraturas de quadril e internados durante o lockdown no Reino Unido.

2. Determinar a mortalidade em 30 dias de pacientes internados com fraturas de quadril.

3. Determinar a mortalidade em 30 dias de pacientes com fratura de quadril e COVID-19.

4. Comparar a mortalidade em 30 dias destes pacientes com dados de anos anteriores. 


\section{Material e Métodos}

Este é um estudo de coorte unicêntrico, observacional e retrospectivo com 65 pacientes com fraturas de quadril internados em nossa instituição.

Os critérios de inclusão foram:

- Pacientes com fraturas do fêmur proximal internados em nossa instituição.

- Pacientes internados entre 23 de março e 22 de maio de 2020.

- Todas as idades.

- Ambos os gêneros.

Os critérios de exclusão foram:

- Fraturas expostas.

- Fraturas patológicas.

- Fraturas periprotéticas.

- Fraturas peri-implantes.

- Politraumatismo.

Os dados coletados foram idade, gênero, tipo de fratura, data de internação, data da cirurgia e tratamento recebido.

Os pacientes foram classificados em quatro categorias conforme sua vulnerabilidade à COVID-19: risco baixo, risco moderado, risco alto e risco muito alto (-Fig. 3 ). ${ }^{9}$

O diagnóstico de COVID-19 foi feito em pacientes com febre e tosse por meio de um teste de reação em cadeia de polimerase (PCR, na sigla em inglês) de amostras de $s w a b$ nasal. Os resultados de PCR foram pesquisados em registros institucionais e da clínica geral.

Como o período de incubação do COVID-19 é de 2 a 14 dias, ${ }^{18}$ os pacientes com resultado positivo em $<2$ dias da internação ou após 14 dias da alta não foram considerados infecções hospitalares.

Os pacientes foram acompanhados por um período mínimo de 30 dias a partir do dia da internação para determinação de mortalidade.

Estes dados de mortalidade foram comparados com os dados do National Hip Fracture Database (NHFD, na sigla em inglês) do Reino Unido. ${ }^{14}$

\section{Resultados}

\section{Dados Demográficos}

Um total de 65 pacientes com fraturas de quadril foi internado em nossa instituição entre 23 de março e 22 de maio.

Destes, 43 eram mulheres (66\%) e 22 eram homens (34\%).

Os pacientes tinham idades entre 63 e 104 anos, com média de 84 anos. Apenas 1 paciente tinha $<65$ anos (63 anos).

No total, 28 eram lesões do lado esquerdo, e 37 do lado direito.

Dos 65 pacientes, 4 foram submetidos ao tratamento conservador, e 61 ao tratamento cirúrgico.

Dos 61 pacientes operados, 34 foram submetidos a hemiartroplastia cimentada (56\%), 21 a fixação com parafuso

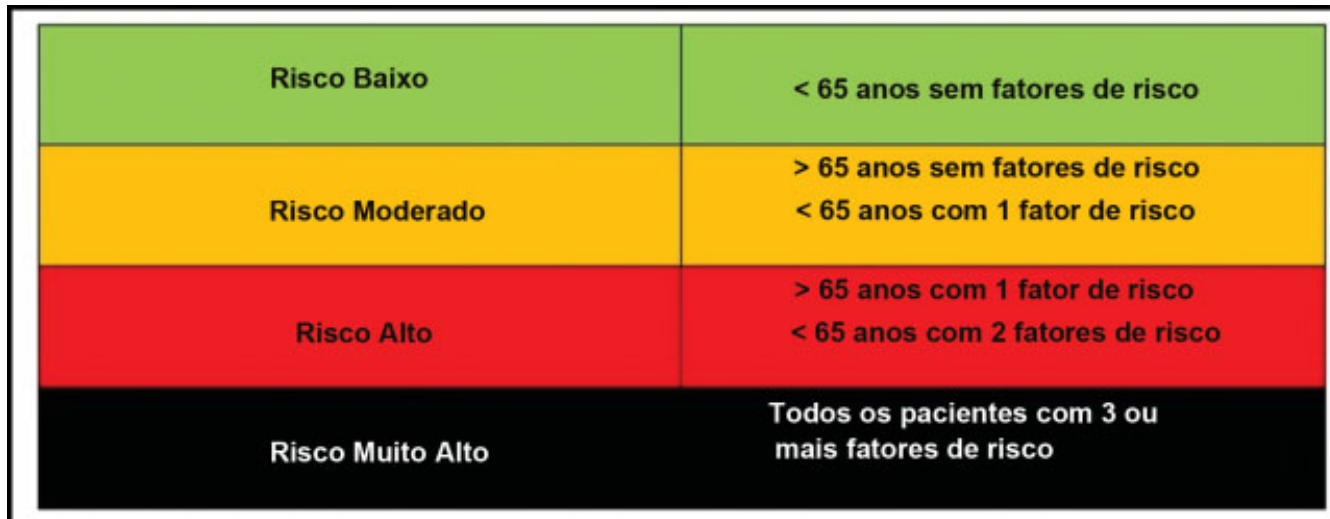

\section{Fatores de Risco Identificados (CDC)}

Asma - Apenas moderada a grave

Doença pulmonar crônica - doença pulmonar obstrutiva crônica, fibrose pulmonar, fibrose cística

Diabetes

Doenças graves - insuficiência cardiaca, doença da artéria coronária,

cardiopatia congênita, cardiomiopatia

Doença renal crônica - Diálise

Obesidade grave - Índice de massa corpórea $(\mathrm{IMC})<40$

Idade - > 65 anos

Comprometimento imunológico - Tratamento de câncer, transplante, inclusive de medula

óssea, deficiência imunológica, HIV com baixo número de CD4+, imunossupressão causada

por medicamentos, inclusive tratamento prolongado com corticosteroides

Doença hepática - Cirrose

Fig. 3 Estratificação de risco de vulnerabilidade à COVID-19 segundo os Centers for Disease Control and prevention (recomendação da British Orthopaedic Association). 
dinâmico de quadril (34\%), e 6, a fixação com haste gama (10\%).

\section{Categoria de Risco}

Os pacientes apresentaram diferentes níveis de risco:

- Nenhum paciente em nossa coorte apresentou risco baixo.

- Quarenta e dois pacientes tinham risco moderado. Destes, 41 pacientes tinham $>65$ anos e não apresentavam comorbidades significativas, enquanto 1 paciente de 63 anos tinha 1 comorbidade.

- Doze pacientes foram classificados como de risco alto. Todos tinham $>65$ anos e 1 comorbidade.

- Onze foram classificados como de risco muito alto, e todos tinham $>65$ anos. Nove tinham duas comorbidades e dois tinham três comorbidades.

A incidência de COVID-19 em pacientes internados com fraturas de quadril após o lockdown no Reino Unido foi:

No total, 10 pacientes foram diagnosticados com COVID19; destes, 3 pacientes foram diagnosticados com COVID-19 durante a internação, e 7 após a alta.

Dos 3 pacientes COVID-positivos durante a hospitalização, 1 foi positivo no $1^{\circ}$ dia de internação, 1 no dia 6 , e 1 no dia 10 .

Daqueles que foram positivos após a alta, 1 foi diagnosticado com COVID-19 no dia da alta, 1 aos 10 dias, 1 aos 12 dias, 1 aos 13 dias, 1 aos 26 dias, e 2 aos 28 dias após a alta.

Não acreditamos que o único paciente com resultado positivo no dia da internação tenha sido infectado no hospital, pois o período mínimo de incubação é de 2 dias; da mesma forma, não consideramos os 3 pacientes com resultado positivo em 26 e 28 dias como infectados no hospital, pois o período máximo de incubação de COVID-19 é de 14 dias.

Assim, consideramos que apenas $6(9 \%)$ pacientes foram infectados durante a sua permanência no hospital.
Mortalidade em 30 dias dos pacientes internados com fraturas de quadril:

Dos 65 pacientes com fraturas de quadril, 10 faleceram em 30 dias (15\%).

Dos 4 submetidos ao tratamento conservador, 2 pacientes faleceram em 30 dias (50\%).

Dos 61 pacientes operados, 8 faleceram em 30 dias (13\%).

Os 10 pacientes que faleceram apresentaram diferentes níveis de risco:

- Sete foram classificados como risco muito alto

- Um paciente foi classificado como risco alto

- Dois pacientes foram classificados como risco médio,

- Nossa coorte não apresentou nenhum paciente de baixo risco.

- Três pacientes dentre os 54 indivíduos com risco moderado e alto faleceram em 30 dias (5,6\%).

Sete dentre os 11 pacientes com risco muito alto faleceram em 30 dias (64\%).

Nove pacientes com COVID-19 foram operados para correção da fratura de quadril; destes, 3 faleceram (33\%). Destes nove pacientes:

- taxa de mortalidade em risco moderado-alto/operado/ COVID +: $1 / 6(17 \%)$

- taxa de mortalidade em risco muito alto/operado/COVID $+: 2 / 3$ (-Fig. 4).

Dos 55 pacientes não infectados por COVID-19, 6 faleceram (11\%). Seis pacientes faleceram durante a internação, $e$ quatro após a alta.

\section{Discussão}

Nosso estudo mostrou que a maioria $(41 / 64 ; 64 \%)$ dos pacientes $>65$ anos não apresentava comorbidade

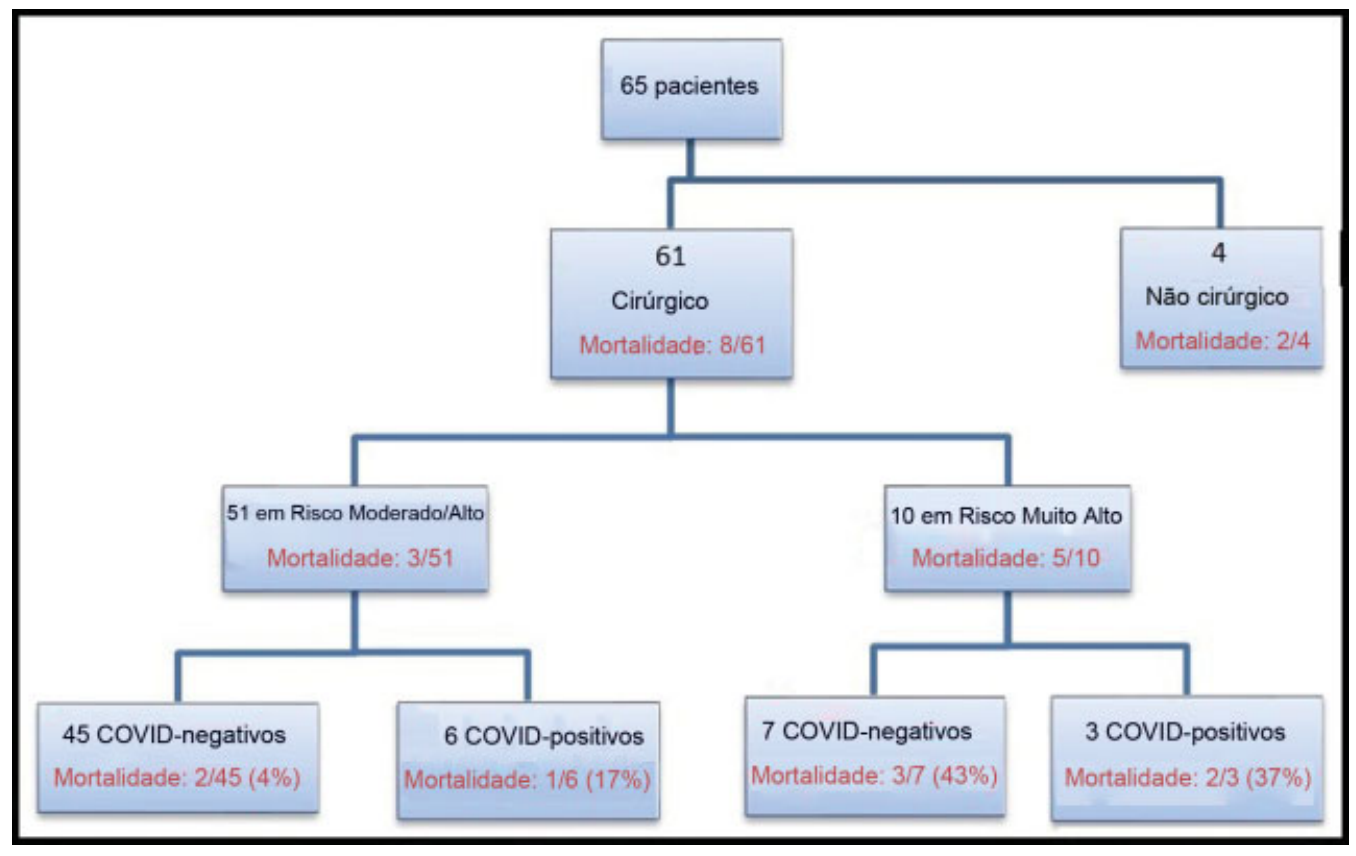

Fig. 4 
Tabela 1 Comparação de estudos

\begin{tabular}{|c|c|c|c|}
\hline & Estudo Atual & Archer et al. ${ }^{16}$ & Muñoz Vives et al. ${ }^{17}$ \\
\hline Período de estudo & $23 / 03 / 2020-22 / 05 / 2020$ & $25 / 03 / 2020-25 / 04 / 2020$ & $14 / 03 / 2020-04 / 04 / 2020$ \\
\hline Tamanho da amostra & 65 (61 operados) & 142(142 operados) & 136 (124 operados) \\
\hline Média de idade (anos) & $84(63-104)$ & $82(55-101)$ & $85(65-101)$ \\
\hline Positivos para COVID-19 & $\begin{array}{l}10 \text { ( } 6 \text { casos adquiridos } \\
\text { no hospital) } \\
15 \%\end{array}$ & $18 ; 13 \%$ & $23 ; 17 \%$ \\
\hline $\begin{array}{l}\text { Mortalidade em } 30 \text { dias } \\
\text { em pacientes } \\
\text { positivos para COVID-19 }\end{array}$ & $40 \%$ & $22,2 \%$ & 30,3\% (mortalidade em 14 dias) \\
\hline $\begin{array}{l}\text { Mortalidade em pacientes } \\
\text { operados não infectados }\end{array}$ & $7,8 \%$ & $4 \%$ & \\
\hline Mortalidade total em 30 dias & $15 \%$ & $6 \%$ & 9,6\% (mortalidade em 14 dias) \\
\hline
\end{tabular}

significativa. Ainda assim, estes indivíduos estavam em risco moderado por causa de sua idade. No total, $18,5 \%$ eram de risco alto e $17 \%$ eram de risco muito alto.

A incidência de infecções nosocomiais por COVID-19 em nossa instituição foi de $9 \%$. A taxa geral de infecção (nosocomial e comunitária) foi de $15 \%$.

A mortalidade geral em 30 dias foi de 15\%. Mesmo em pacientes com fraturas de quadril, mas sem COVID-19, a mortalidade em 30 dias foi de $9 \%$. Este número é maior do que a média nacional de 6,2 de acordo com o NHFD. ${ }^{14}$

A mortalidade foi muito maior em pacientes COVIDpositivos, de $40 \%$.

$\mathrm{O}$ achado mais importante, porém, foi a mortalidade em 30 dias de pacientes com risco moderado e até alto (85\% dos pacientes) de apenas 5,6\%, que é inferior à média do Reino Unido.

Nos pacientes de risco muito alto operados nesse período, o risco de mortalidade foi de $63 \%$.

Um estudo semelhante, com 142 pacientes, feito por Archer et al. no Reino Unido, observou uma incidência de COVID-19 em pacientes internados com fraturas de quadril de $12,67 \%$ e mortalidade em 30 dias em pacientes com fratura do colo do fêmur e COVID-19 de $22,2 \%{ }^{16}$

Outro estudo com 136 pacientes com fratura de quadril na Espanha descobriu que a incidência de COVID-19 era de $17 \%$. A taxa de mortalidade total em 14 dias foi de $9,6 \%$, enquanto a taxa de mortalidade em 14 dias em pacientes COVID-19positivos foi de $30,4 \%{ }^{17}$

Estes dois os estudos usaram a classificação da American Society of Anesthesiologists (ASA, na sigla em inglês) ao invés da classificação do Centers for Disease Control and Prevention (CDC, na sigla em inglês) para a avaliação de risco (-Tabela 1).

Se combinarmos os dados dos três estudos, de 343 pacientes admitidos com fraturas de quadril, 51 foram infectados com COVID-19 (15\%). A mortalidade em pacientes com COVID-19 foi de $29 \%$. A mortalidade total de curto prazo de fraturas de quadril durante este período foi $>9,3 \%$.

\section{Conclusão}

Nosso estudo mostra a vulnerabilidade da população idosa com comorbidades submetida a cirurgias durante a pior fase da pandemia de COVID-19. A mortalidade em 30 dias de pacientes com fraturas de quadril parece ter aumentado com o início da pandemia. Os pacientes mais atingidos são aqueles na categoria de risco muito alto e aqueles com diagnóstico de COVID-19.

No entanto, a mortalidade em pacientes com risco moderado e até alto foi de apenas 5,6\%, o que é inferior à média nacional de tempos não pandêmicos. Este grupo constitui $85 \%$ da população de pacientes idosos.

Somos da opinião que, como o pico já passou, o reinício da realização de cirurgias eletivas em pacientes com risco baixo, moderado e alto deve ser seguro, sem um aumento apreciável na mortalidade. Acreditamos que há necessidade de mais dados e mais tempo antes do reinício de cirurgias eletivas em pacientes de risco muito alto.

Recomendamos estudos futuros para análise da mortalidade em pacientes de risco muito alto no período após o pico de COVID (a partir de junho de 2020) e sua comparação à mortalidade anterior no grupo de pacientes de risco muito alto. Também recomendamos um estudo mais amplo de mortalidade em pacientes de risco baixo, moderado e alto que foram operados e contraíram COVID-19.

\section{Suporte Financeiro}

Não houve suporte financeiro de fontes públicas, comerciais, ou sem fins lucrativos.

Conflito de Interesses

Os autores não têm conflitos de interesse a declarar

\section{Referências}

1 Hui DSI, , I Azhar E, Madani TA, et al. The continuing 2019-nCoV epidemic threat of novel coronaviruses to global health - The latest 2019 novel coronavirus outbreak in Wuhan, China. Int J Infect Dis 2020;91:264-266 
600 Mortalidade e Incidência de SARS-CoV-2 (COVID-19) Shah et al.

2 "Coronavirus disease 2019 (COVID-19)-Symptoms and causes". Mayo Clinic Retrieved 14 April 2020. Available from: https:// www.mayoclinic.org/diseases-conditions/coronavirus/symptoms-causes $/$ syc -20479963 ? page $=0 \&$ citems $=10 \& \mathrm{p}=1$

3 Moss P, Barlow G, Easom N, Lillie P, Samson A. Lessons for managing high-consequence infections from first COVID-19 cases in the UK. Lancet 2020;395(10227):e46

4 "WHO Director-General's opening remarks at the media briefing on COVID-19". World Health Organization (WHO) (Press release) 11 March 2020. Archived from the original on 11 March 2020. Available from: https://www.who.int/dg/speeches/detail/whodirector-general-s-opening-remarks-at-the-media-briefing-oncovid-19-11-march-2020

5 "PM announces strict new curbs on life in UK". BBC News 23 March 2020. Retrieved 23 March 2020

6 Cases in United Kingdom. Available from: https://coronavirusstaging.data.gov.uk/cases

7 Shaun Lintern (Health Correspondent) Coronavirus: Routine NHS operations cancelled in effort to free up 30,000 hospital beds, Independent, Tuesday 17 March 2020 15:46. Available from: https://www.independent.co.uk/news/health/coronavirus-uk-update-cases-nhs-beds-operations-latest-a9406966. html

8 Ian Sample. More than $2 \mathrm{~m}$ operations cancelled as NHS fights Covid-19, Guardian, 26 April 2020. Available from: https://www. theguardian.com/society/2020/apr/26/more-than-two-millionoperations-cancelled-as-nhs-fights-covid-19

9 Re-starting non-urgent trauma and orthopaedic care: Full guidance, British Orthopaedic Association. Available from: boa.ac. uk/uploads/assets/9383a53f-36d8-4782-8fe264c691b39b15/ BOA-Guidance-for-restart-full-doc-final2-v11.pdf
10 Lei S, Jiang F, Su W, et al. Clinical characteristics and outcomes of patients undergoing surgeries during the incubation period of COVID-19 infection. [published online ahead of print, 2020 Apr 5] EClinicalMedicine 2020;21:100331

11 People Who Are at Increased Risk for Severe Illness. CDC, Updated June 25, 2020. Available from: https://www.cdc.gov/coronavirus/ 2019-ncov/need-extra-precautions/people-at-increased-risk.html

12 National Hip Fracture Database Annual Report 2018. Available from: https://data.gov.uk/dataset/3a1f3c15-3789-4299-b24bcd0a5b1f065b/national-hip-fracture-database-annual-report-2018

13 Edelmuth SVCL, Sorio GN, Sprovieri FAA, Gali JC, Peron SF Comorbidities, clinical intercurrences, and factors associated with mortality in elderly patients admitted for a hip fracture. Rev Bras Ortop 2018;53(05):543-551

14 National Hip Fracture Database. Available from: https://www. nhfd.co.uk/20/nhfdcharts.nsf/vwCharts/OverallPerformance

15 Downey C, Kelly M, Quinlan JF. Changing trends in the mortality rate at 1 -year post hip fracture - a systematic review. World J Orthop 2019;10(03):166-175

16 Archer JE, Kapoor S, Piper D, et al. The impact of COVID-19 on 30day mortality in patients with neck of femur fractures. Bone Joint Open 2020;1(07):326-329

17 Muñoz Vives JM, Jornet-Gibert M, Cámara-Cabrera J, et al; Spanish HIP-COVID Investigation Group. Mortality Rates of Patients with Proximal Femoral Fracture in a Worldwide Pandemic: Preliminary Results of the Spanish HIP-COVID Observational Study. J Bone Joint Surg Am 2020;102(13):e69

18 European Centre for Disease Prevention and Control (ECDC) Q \& A on novel coronavirus. Stockholm: ECDC; 2020. [Accessed 4 Feb 2020]. Available from: https://www.ecdc.europa.eu/en/novelcoronavirus-china/questions-answers 